Departamento de Ciencias Neurológicas Oriente, Facultad de Medicina Universidad de Chile. Servicio de Neurología, Hospital del Salvador, Santiago, Chile.

Recibido el 15 de junio de 2016, aceptado el 16 de diciembre de 2016.

Correspondencia a: Dr. Luis Cartier Rovirosa Avenida Salvador 364, Servicio de Neurología HDS, Providencia, Santiago. Icartier@med.uchile.cl

\section{Retracción palpebral de causa neurológica. Presentación de tres pacientes, interpretación patogénica}

\author{
LUIS CARTIER R., JORGE GUZMÁN S., RENZO PASQUALI F.
}

Eyelid retraction, has received limited attention and it has passively been interpreted as the result of an overactive levator palpebrae superioris muscle secondary to midbrain injury. However, eyelid retractions can occur in other neurological diseases, not directly related with the midbrain. We report three patients who developed eyelid retraction. One patient had a bilateral eyelid retraction, related with Creutzfeldt-Jakob disease (CJD). Another patient had a unilateral right eyelid retraction associated with a thalamic-mesencephalic infarct. The third patient had a bilateral pontine infarction on magnetic resonance imaging. In the patient with CJD, eyelid retraction did not subside. Among patients with infarctions, the retraction persisted after focal symptoms had subsided, showing an evolution that was apparently independent of the basic process. The analysis of these patients allows us to conclude that the pathogenesis of eyelid retraction includes supranuclear mechanisms in both the development and maintenance of the phenomenon. Unilateral or bilateral eyelid retraction does not alter the normal function of eyelid, which ever had normal close eye blink. In these reported cases, a hyperactivity of levator palpebrae superioris muscle was clinically ruled out.

(Rev Med Chile 2017; 145: 264-268)

Key words: Creutzfelt-Jakob Syndrome; Eyelid Diseases; Mesencephalon; Nervous system Diseases; Ocular Physiological Phenomena.

L a amplitud de la apertura ocular depende de la activación del elevador de los párpados y de la inhibición del orbicular. Los músculos elevadores son inervados desde un núcleo mesencefálico único, por el III par correspondiente. El orbicular es inervado por el facial. El tono de la apertura palpebral la controla el músculo de Müller, de inervación simpática ${ }^{1}$.

Los núcleos oculomotores están ubicados en el mesencéfalo por delante del acueducto de Silvio, donde el núcleo de los elevadores tiene una ubicación central y posterior, próxima al acueducto. La coordinación óculo-palpebral se realiza en estructuras subyacentes, en los núcleos intersticiales mediales interconectados por la comisura posterior y también con los núcleos mesencéfalo-protuberanciales, a través de los haces longitudinales mediales. Estas estructuras intermedias regulan la coordinación y sincronía entre elevadores y músculos oculomotores, permitiendo adecuar la disposición palpebral en las miradas verticales u otras ${ }^{1}$.

El párpado superior cubre parcialmente el iris en la apertura normal del ojo, sin embargo, en condiciones patológicas el iris queda completamente descubierto debido a una sobreelevación del párpado superior, constituyendo lo que se ha identificado como: síndrome de retracción 
palpebral, el que fue descrito por Collier en $1927^{2}$ en un paciente portador de un infarto tálamo-mesencefálico.

Esta sobreelevación del párpado superior puede tener causas neurológicas (síndrome Collier) ${ }^{2}$, endocrinas (oftalmopatías de la enfermedad de Graves) ${ }^{3}$, infecciosas (lepra) ${ }^{4}$, inflamatorias (oftalmopatías inmunes) ${ }^{5,6}$, entre otras causas (miastenia, etc. $)^{7,8}$. Analizaremos específicamente la patogenia de la sobreelevación palpebral de origen neurológico que, teniendo distintas causas, destacan por su frecuencia las de etiología vascular.

Basados en los hallazgos histopatológicos de la comunicación de Collier, autores posteriores $^{9,10}$ han interpretado que la sobreelevación palpebral por lesiones vasculares del cerebro sería consecuencia de una hiperactividad del elevador (retracción), causada por lesiones en la comisura posterior mesencefálica ${ }^{11}$. Sin embargo, en los pocos pacientes analizados, se ha observado que mantienen su capacidad de pestañeo y de oclusión ocular, sin la restricción que una hiperactividad del elevador generaría. Este hecho, unido a la hiperapertura ocular de pacientes que no tienen lesiones mesencefálicas focales, como la enfermedad de Parkinson o la enfermedad de Creutzfeldt-Jakob (ECJ), han hecho necesario postular una nueva interpretación patogénica de la sobreelevación palpebral.

Presentamos tres enfermos con retracción palpebral que fueron seguidos clínicamente, un paciente con enfermedad de Creutzfeldt-Jakob y dos con lesiones vasculares.

\section{Pacientes}

\section{Enfermedad de Creutzfeldt-Jakob}

Paciente de 53 años que a fines de marzo del 2008 notó mayor fatigabilidad, fallas de memoria e insomnio. Recibió terapia antidepresiva que no modificó su condición. La sensación de fatiga llegó a ser permanente, así como el insomnio, apareciendo inestabilidad de la marcha, lateropulsiones y temblor de las manos. Luego se agregó visión borrosa y se consolidó su incapacidad de marcha. Ingresó a Neurología con una "mirada hierática" (ojos muy abiertos), debida a una sobreelevación palpebral bilateral . La paciente estaba vigilante, atenta, orientada, lenguaje conservado, sin alteraciones práxicas, fondo de ojo y campos visuales normales, agudeza visual disminuida; tenía tem- blor de extremidades superiores y la bipedestación desencadenaba temblor en las piernas y el tronco, haciéndole imposible la marcha; hiperreflexia osteotendinea generalizada, sin Babinski, ni alteraciones de la sensibilidad. Se apreciaba eutrófica, sin hallazgos en la exploración clínica general. Hemograma, pruebas hepáticas, electrolitos y TSH normales, líquido cefalorraquídeo normal. Antitiroglobulina $621 \mathrm{UI} / \mathrm{ml}$ y antitiroperoxidasa $845 \mathrm{UI} / \mathrm{ml}$. Resonancia magnética (RM) de cerebro en T2 y FLAIR mostraba hiperintensidad de los núcleos basales (Figura 1). Electroencefalograma (EEG) con actividad lenta inespecífica. Se postuló enfermedad de Creutzfeldt-Jakob o encefalopatía de Hashimoto. Aunque tenía elevada la proteína 14-3-3, igualmente se trató con tres bolos de metilprednisolona, que no la mejoraron, manteniéndose la sobreelevación palpebral. Seguidamente, se definió una ceguera cortical, sin embargo, mantenía la apariencia de ver con los ojos muy abiertos. Su hablar se hizo incomprensible, asociando disartria, afasia y daño cognitivo. Finalmente, llegó a la postración, aparecieron

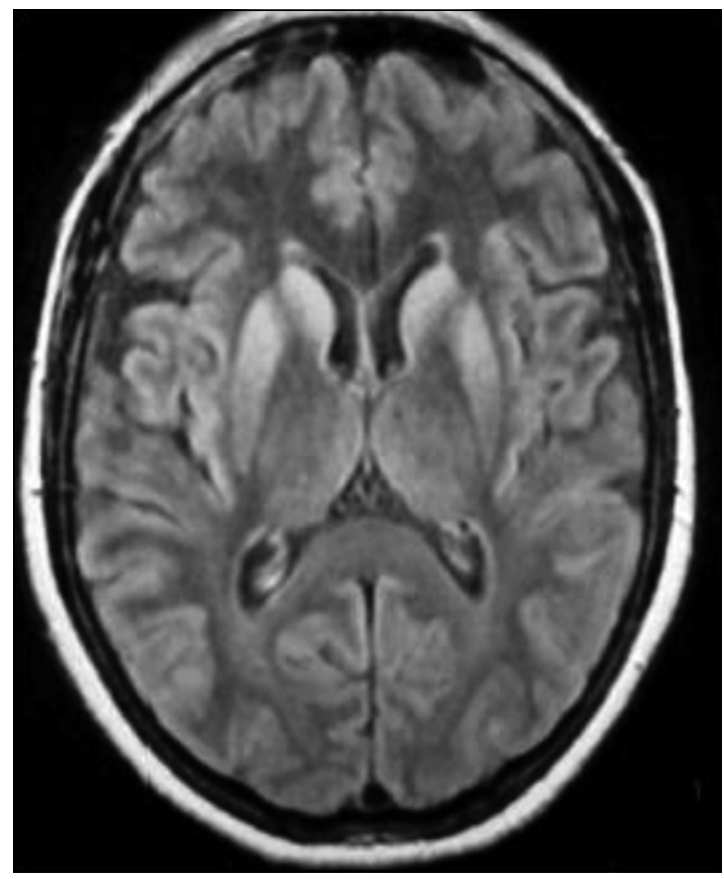

Figura 1. Corte axial de RM en secuencia FLAIR de la paciente $N^{\circ} 1$, con enfermedad de Creutzfeldt-Jakob. Se observa hiperintensidad putaminal y de los núcleos caudados. También hiperintensidad de la circunvolución límbica en forma bilateral y de la ínsula derecha. 
mioclonías en las extremidades superiores y en la cara, el EEG de control confirmó la ECJ. Falleció en octubre por una neumonía intrahospitalaria, después de siete meses de enfermedad.

\section{Infarto tálamo-mesencefálico}

Enferma de 35 años, operada de una comunicación interventricular diez años antes, quedó con marcapaso desde entonces. Inició su enfermedad con una cefalea frontal progresiva, náuseas y fotopsias; súbitamente presentó pérdida de conciencia, contracción tónica generalizada, seguida de clonías rítmicas de las extremidades. Cuando recuperó la conciencia tenía visión doble. Trasladada a Urgencia, ingresó vigilante, parcialmente orientada, lenguaje normal, pupilas iguales, agudeza visual conservada, retracción del párpado superior derecho y paresia del sexto par derecho, ojo izquierdo hipotrópico y leve paresia braquio-facial derecha sin Babinski ni alteraciones sensitivas o cerebelosas. Tomografía computada (TAC) cerebral, exámenes y líquido cefalorraquídeo normales, VDRL no reactivo. Estuvo somnolienta los primeros días, la TAC de control mostró hipodensidad medial en el tálamo izquierdo prolongada al mesencéfalo (Figura 2). Al quinto día había desaparecido la paresia braquial, la hipotropía del ojo izquierdo y la dificultad para abducir el ojo derecho. Evaluada por cardiología, se estableció que el agotamiento del generador del marcapaso originó una arritmia paroxística que causó el infarto tálamo-mesencefálico. Al alta se había recuperado de todas las alteraciones neurológicas, salvo la retracción del párpado superior derecho que se mantenía al décimo día.

\section{Infarto protuberancial}

Mujer de 27 años, sin antecedentes mórbidos, concurre a Urgencia por cefalea occípito-cervical progresiva, diplopía, parálisis facial izquierda y adormecimiento corporal derecho. Al ingreso estaba cuadriparética, vigilante y orientada, muy disártrica, pupilas iguales reactivas, mayor apertura ocular derecha, paresia del sexto par izquierdo y parálisis facial periférica izquierda. Angio-TAC de vasos del cuello y cerebrales mostraron oclusión de la arteria vertebral izquierda y de los segmentos V3-V4 del tronco basilar. La RM mostró un infarto bilateral de la protuberancia y del bulbo raquídeo y cerebelo izquierdo (Figura 3). Se desarrolló síndrome de enclaustramiento, (tetraplejia hipotónica arrefléctica, Babinski bilateral), parálisis facial periférica izquierda y retracción del párpado superior derecho, tenía limitada las miradas laterales y mantenía la mandíbula caída, sin poder cerrar la boca. Siempre se mantuvo vigilante, comprendía y

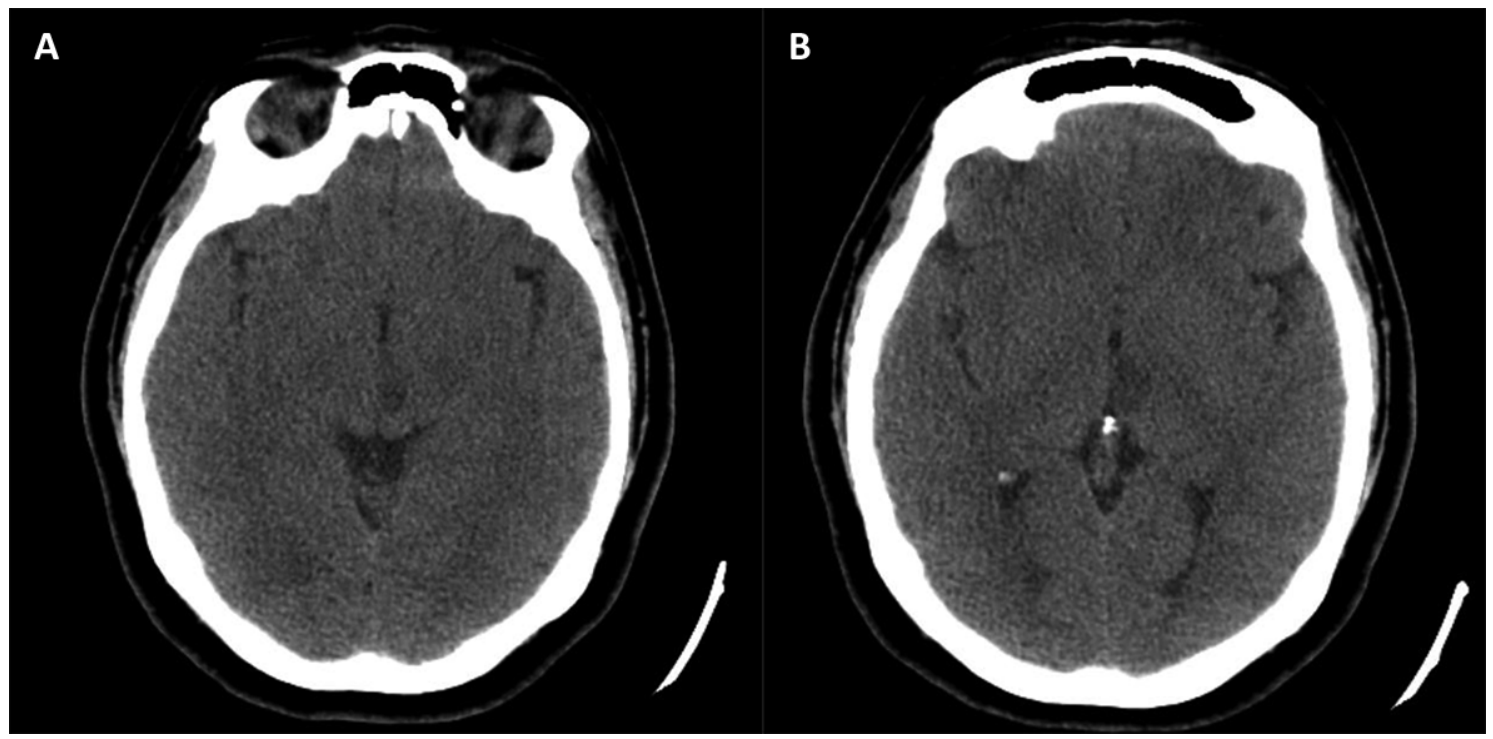

Figura 2. Tomografía axial computarizada de la paciente $N^{\circ} 2$. En A) se observa hipodensidad mesencefálica derecha y en B) hipodensidad medial del tálamo izquierdo, compatible con una lesión isquémica tálamo-mesencefálica. 

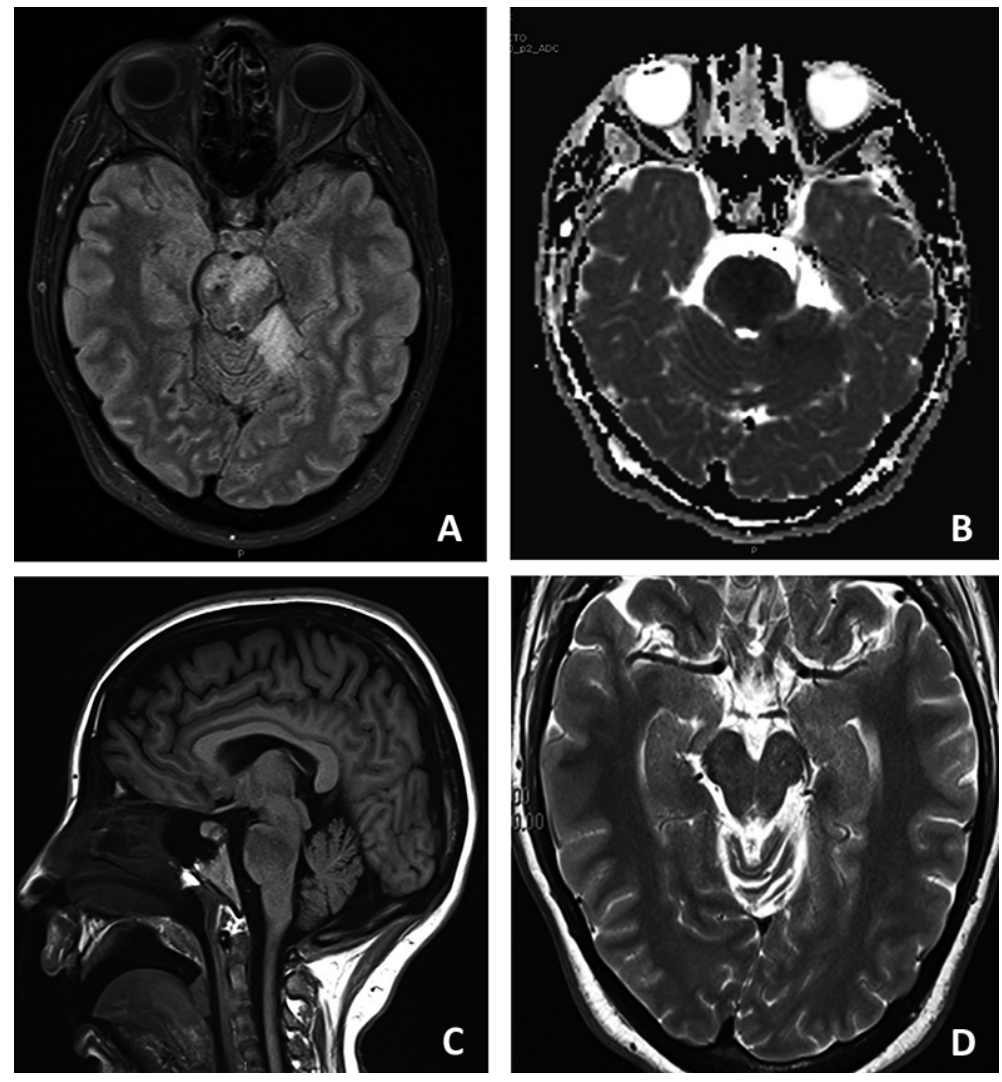

Figura 3. Resonancia magnética de cerebro de la paciente $\mathrm{N}^{\circ} 3$. Cortes axiales de la protuberancia y mesencéfalo en A) secuencia FLAIR que muestra hiperintensidad del centro y parte anterior de la protuberancia e hiperintensidad sectorial del cerebelo izquierdo; en B) Mapa ADC del mismo corte; en C) Corte sagital del encéfalo donde se aprecia la lesión protuberancial y la indemnidad del cerebro; en D) Corte en secuencia T2 del mesencéfalo sin lesiones. Imágenes compatibles con una disección vertebral y la oclusión parcial de la arteria basilar.

respondía preguntas de si o no, ocluyendo los ojos, no movía la cabeza. Después de tres semanas empezaron a reaparecer los reflejos osteotendíneos, mejoró la oculomotilidad horizontal, sin embargo, el síndrome de enclaustramiento y la retracción palpebral se mantenían. A las seis semanas podía cerrar la boca y la parálisis facial fue remplazada por un espasmo facial tónico, se mantenía la cuadriplejia y la retracción palpebral derecha.

\section{Discusión}

Experimentalmente se ha demostrado que la apertura ocular se puede activar estimulando áreas de la corteza prefrontal ${ }^{12}$. Esta relación córtico-palpebral tiene su expresión fisiológica en la apertura de los ojos al despertar, que sugiere conexiones retículo-corticales permanentes. La hiperapertura ocular es un fenómeno extraordinario y habitualmente expresa estereotipos de origen córtico-subcortical. Para entender la sobreelevación de los párpados en situaciones patológicas es interesante analizar la reacción de sobresalto que partiendo en la substancia reticular del puente activa respuestas estructuradas en la corteza cerebral. Se presume que el sobresalto tiene un origen principalmente acústico. Experimentalmente, se ha visto que un súbito estímulo sonoro activa las neuronas auditivas ubicadas en el puente, que sin transición activan las grandes neuronas reticulares de la protuberancia, dando origen al sobresalto. Se expresa con oclusión o hiperapertura palpebral, inclinación de la cabeza y paralogización temporal del cuerpo ${ }^{13}$. Otro ejemplo es la reacción de sorpresa que identificamos por frases como: "se le salieron los ojos", "se quedó con la boca abierta", o "se cayó de espaldas". En este caso, tanto la hiperapertura ocular, así como las otras manifestaciones motoras, parecen expresar un estereotipo supranuclear inhibitorio. Lo mismo vale para el espasmo ocular, que de modo azaroso y súbito instala una oclusión palpebral completa y una incapacidad transitoria para abrir los ojos. Este síndrome fue interpretado inicialmente como 
una diskinesia extrapiramidal ${ }^{14}$, pero el estudio y seguimiento de enfermos pudo demostrar que el espasmo ocular bilateral tenía su punto de partida en lesiones unilaterales de la corteza cerebral o de otras estructuras encefálicas ${ }^{15}$. La sobreelevación palpebral en la enfermedad de Creutzfeldt-Jakob, o "mirada hierática", suele asociarse a ceguera cortical, indudablemente originada en el compromiso de la corteza occipital, donde existen áreas reconocidas, cuyo estímulo experimental produce apertura ocular ${ }^{12}$. Esta sobreelevación palpebral en la ECJ tiene además un valor clínico-diagnóstico semejante al de las mioclonías ${ }^{16}$. La retracción palpebral unilateral de origen vascular, de acuerdo a algunos autores ${ }^{9}$, tendría su origen en una lesión de los núcleos intersticiales o de la comisura posterior que rodea el acueducto, ambas estructuras están muy relacionadas con la substancia gris periacueductal, cuya estimulación experimental produce la sobreelevación unilateral del párpado correspondiente ${ }^{1}$. Además resulta lícito pensar que la sobreelevación palpebral no sólo es producto de un presunto desenfreno del elevador, sino que participa también la natural inhibición refleja del orbicular y en algunos casos una sobreinhibición del orbicular, como en la expresión de sorpresa, de origen cortical.

En los casos de la literatura ${ }^{2,9-11}$ y en los nuestros, la retracción palpebral se mantuvo después de la mejoría parcial o total del síndrome original, sugiriendo que dicha persistencia establece que las lesiones sólo serían mecanismos inductores. Es interesante resaltar la paciente del caso 3, que teniendo una lesión limitada a la protuberancial en la RM, desarrolló una retracción palpebral derecha persistente, aparentemente inexplicable por la sola lesión protuberancial.

La llamada retracción palpebral sigue siendo un fenómeno peculiar y estudiado insuficientemente, que no pude ser atribuido sólo a hiperactividad del elevador.

\section{Referencias}

1. Schmidtke K, Buttner-Ennever. Nervous control of eyelid function a review of clinical, experimental and pathological data. Brain 1992; 115: 227-47.

2. Collier J. Nuclear ophthalmoplegia, with especial reference to retraction of the lids and ptosis and to lesions of the posterior commissure. Brain 1927; 50: 488-98.
3. Gadipatti R V. Eyelid retraction, lid lag, lagophthalmos, and von Graefe's sign: quantifying the eyelid features of Graves' ophthalmopathy. Ophthalmology 2008; 115 : 1083-5.

4. Ebenezer D, Shirley CH, Sigamoni A. Lid retraction as an indicator of lagophthalmos in leprosy; a preliminary report. Intern. J. Leprosy 2014; 62: 436.

5. Tani J, Gopinath B, Nguyen B, Wall JR. Extraocular muscle autoimmunity and orbital fat inflammation in thyroid-associated ophthalmopathy. Exp Rev Clin Immunol 2007; 3: 299-311.

6. Gopinath B, Ma G, Lahooti H, Wall JR. A Case of Hashimoto's thyroiditis presenting with chronic upper eye lid retraction and positive calsequestrin and collagen XIII Antibodies. International J Endocrino Metabolism 2008; 6: 34-7.

7. Pedroso JL, Sgobbi de Souza PV, Vieira de Rezende Pinto WB, Albuquerque MV, Barsottini OG. Eyelid retraction is not a pathognomonic sign of Machado-Joseph disease in the context of spinocerebellar ataxias. Arq Neuro-Psiquiatr 2014; 72.

8. Khwaja GA, Batla A, Gupta M, Cowdhury D, Ranjan R. Unilateral ptosis and contralateral lid retraction. An unusual sign in recurrent attacks of ocular myasthenia gravis. JIACM 2013; 14: 273-7.

9. Galleta SL, Gray LG, Raps EC, Grossman RI, Schatz NS. Unilateral ptosis and contralateral eyelid retraction from a thalamic-midbrain infartaction. J. Clinical Neuro-Ophthalmology 1993; 13: 221-4.

10. Gaymard B, Larmande P, De Toffol B, Autret A. Reversible nuclear oculomotor nerve paralysis, caused by a primary mesencephalic hemorrage. Eur Neurol 1990; 30: $128-31$.

11. Gaymard B, Lafitte C, Gelot A, De Toffol B. Plus-minus lid syndrome. J Neurol Neurosurg and Psychiatry 1992; 55: 846-8.

12. Caplan LR. Ptosis. J Neurol Neurosurg Psychiatry 1974; 37: 1-7.

13. Koch M. The neurobiology of startle. Progress in Neurobiology 1999; 59: 107-28.

14. Georgescu D, Vagefi MR, Mcmullan TF, Mccann JD, ANDERSON RL. Upper eyelid myectomy in blepharospasm with associated apraxia of lid opening. Am J Ophthalmol 2008;145: 541-7.

15. Khooshnoodi MA, Factor SA, Jinnah HA. Secondary blepharospasm associated with structural lesions of the brain. J Neurol Sci; 2013: 331: 98-101.

16. Cartier L, Quiroz G, Leiva M, Vergara C. Identificación clínica y patológica de las diversas formas de la enfermedad de Creutzfeldt Jakob en Chile. Rev Med Chile 2012; 140: $161-8$. 